\title{
SCHWARZ-PICK-TYPE ESTIMATES FOR THE HYPERBOLIC DERIVATIVE
}

\author{
PETER R. MERCER
}

Received 12 February 2005; Revised 3 November 2005; Accepted 8 November 2005

We obtain Schwarz-Pick-type estimates for the hyperbolic derivative of an analytic selfmap of the unit disk in $\mathbb{C}$.

Copyright (c) 2006 Peter R. Mercer. This is an open access article distributed under the Creative Commons Attribution License, which permits unrestricted use, distribution, and reproduction in any medium, provided the original work is properly cited.

\section{Preliminaries}

We denote by $\Delta$ the open unit disk in $\mathbb{C}$, and for $z \in \Delta$, we denote by $\phi_{z} \in \operatorname{Aut}(\Delta)$ the automorphism which interchanges 0 and $z: \phi_{z}(\lambda)=(z-\lambda) /(1-\bar{z} \lambda)$. We denote by $\rho$ the hyperbolic distance on $\Delta$ :

$$
\rho(\lambda, z)=\tanh ^{-1}\left|\phi_{z}(\lambda)\right|=\frac{1}{2} \log \frac{1+\left|\phi_{z}(\lambda)\right|}{1-\left|\phi_{z}(\lambda)\right|} .
$$

The following is a well-known consequence of the maximum principle.

Schwarz's Lemma 1.1. Let $f: \Delta \rightarrow \Delta$ be analytic with $f(0)=0$. Then

$$
|f(\lambda)| \leq|\lambda|, \quad \text { that is, } \rho(f(\lambda), f(0)) \leq \rho(\lambda, 0) \forall \lambda \in \Delta \text {. }
$$

Consequently, we have also $\left|f^{\prime}(0)\right| \leq 1$. To remove the normalization $f(0)=0$, one may consider the function

$$
g=\phi_{f(z)} \circ f \circ \phi_{z}
$$

which has

$$
g(0)=0, \quad g^{\prime}(0)=\frac{f^{\prime}(z)\left(1-|z|^{2}\right)}{1-|f(z)|^{2}}
$$

to obtain the following.

Hindawi Publishing Corporation Journal of Inequalities and Applications Volume 2006, Article ID 96368, Pages 1-6 DOI 10.1155/JIA/2006/96368 
2 Schwarz-Pick-type estimates for the hyperbolic derivative

Schwarz-Pick Lemma 1.2. Let $f: \Delta \rightarrow \Delta$ be analytic. Then,

$$
\left|\phi_{f(z)} \circ f(\lambda)\right| \leq\left|\phi_{z}(\lambda)\right|, \quad \text { that is, } \rho(f(\lambda), f(z)) \leq \rho(\lambda, z) \forall \lambda, z \in \Delta \text {. }
$$

Consequently, $f^{*}(z):=g^{\prime}(0)$ has $\left|f^{*}(z)\right| \leq 1$, and so $\rho\left(f^{*}(z), \cdot\right)$ is defined on $\Delta$, as long as $f$ is not an automorphism-for in this case, $\left|f^{*}\right| \equiv 1$. As such, we are interested in the following two results.

Theorem 1.3 (see [6]). Let $f: \Delta \rightarrow \Delta$ be analytic, and not an automorphism. Then

$$
\left|\rho\left(0, f^{*}(\lambda)\right)-\rho\left(0, f^{*}(z)\right)\right| \leq 2 \rho(\lambda, z) \quad \forall \lambda, z \in \Delta .
$$

So, for example, if $f^{*}(\lambda)$ and $f^{*}(z)$ are on the same side of a ray emanating from the origin, then $\rho\left(f^{*}(\lambda), f^{*}(z)\right) \leq 2 \rho(\lambda, z)$.

Theorem 1.4 (see [1]). Let $f: \Delta \rightarrow \Delta$ be analytic, not an automorphism, with $f(0)=0$. Then

$$
\rho\left(f^{*}(0), f^{*}(z)\right) \leq 2 \rho(0, z) \quad \forall z \in \Delta .
$$

In the next section of this paper, we employ a procedure which yields simple proofs of Theorems 1.3 and 1.4 and extends these results. In particular, Theorem 1.4 is not applicable if $f(0) \neq 0$, as the function $\exp ((\lambda+1) /(\lambda-1))$ shows. Below however, we obtain a version (Proposition 2.3) which removes the normalization and applies at any pair of points in $\Delta$, thus furnishing a more complete analog of Schwarz-Pick Lemma 1.2 for $f^{*}$. In the final section, we obtain some further related results.

We will use the following easily verified facts.

(A) Schwarz-Pick Lemma 1.2 and a little manipulation reveal that $f(\lambda)$ lies in the closed disk with center $c=f(z)\left(1-\left|\phi_{z}(\lambda)\right|^{2}\right) /\left(1-|f(z)|^{2}\left|\phi_{z}(\lambda)\right|^{2}\right)$ and radius $r=\left|\phi_{z}(\lambda)\right|\left(1-|f(z)|^{2}\right) /\left(1-|f(z)|^{2}\left|\phi_{z}(\lambda)\right|^{2}\right)$. Consequently, $|c|-r \leq|f(\lambda)| \leq$ $|c|+r$. That is,

$$
\frac{|f(z)|-\left|\phi_{z}(\lambda)\right|}{1-|f(z)|\left|\phi_{z}(\lambda)\right|} \leq|f(\lambda)| \leq \frac{|f(z)|+\left|\phi_{z}(\lambda)\right|}{1+|f(z)|\left|\phi_{z}(\lambda)\right|} .
$$

(B) For $x \in[0,1],(t+x) /(1+t x)$ and $(t-x) /(1-t x)$ are increasing functions of $t \in$ $[0,1]$.

(C)

$$
\left(1+\frac{(y+x) /(1+y x)+x}{1+((y+x) /(1+y x)) x}\right) \div\left(1-\frac{(y+x) /(1+y x)+x}{1+((y+x) /(1+y x)) x}\right)=\frac{1+y}{1-y}\left(\frac{1+x}{1-x}\right)^{2} .
$$

(D)

$$
\left(1+\frac{(y-x) /(1-y x)-x}{1-((y-x) /(1-y x)) x}\right) \div\left(1-\frac{(y-x) /(1-y x)-x}{1-((y-x) /(1-y x)) x}\right)=\frac{1+y}{1-y}\left(\frac{1-x}{1+x}\right)^{2}
$$




\section{Results}

We see below that the following has Theorem 1.3 as a consequence.

Proposition 2.1. Let $f: \Delta \rightarrow \Delta$ be analytic. Then for all $z_{1}, z_{2} \in \Delta$,

$$
\begin{aligned}
& \frac{\left(\left|f^{*}\left(z_{1}\right)\right|-\left|\phi_{z_{1}}\left(z_{2}\right)\right|\right) /\left(1-\left|f^{*}\left(z_{1}\right)\right|\left|\phi_{z_{1}}\left(z_{2}\right)\right|\right)-\left|\phi_{z_{1}}\left(z_{2}\right)\right|}{1-\left(\left|f^{*}\left(z_{1}\right)\right|-\left|\phi_{z_{1}}\left(z_{2}\right)\right|\right) /\left(1-\left|f^{*}\left(z_{1}\right)\right|\left|\phi_{z_{1}}\left(z_{2}\right)\right|\right)\left|\phi_{z_{1}}\left(z_{2}\right)\right|} \\
& \quad \leq\left|f^{*}\left(z_{2}\right)\right| \leq \frac{\left(\left|f^{*}\left(z_{1}\right)\right|+\left|\phi_{z_{1}}\left(z_{2}\right)\right|\right) /\left(1+\left|f^{*}\left(z_{1}\right)\right|\left|\phi_{z_{1}}\left(z_{2}\right)\right|\right)+\left|\phi_{z_{1}}\left(z_{2}\right)\right|}{1+\left(\left(\left|f^{*}\left(z_{1}\right)\right|+\left|\phi_{z_{1}}\left(z_{2}\right)\right|\right) /\left(1+\left|f^{*}\left(z_{1}\right)\right|\left|\phi_{z_{1}}\left(z_{2}\right)\right|\right)\right)\left|\phi_{z_{1}}\left(z_{2}\right)\right|} .
\end{aligned}
$$

Proof. For $f: \Delta \rightarrow \Delta$ analytic, we fix $w_{1}=f\left(z_{1}\right), w_{2}=f\left(z_{2}\right)$ and set

$$
g=\left(\phi_{w_{2}} \circ f\right) / \phi_{z_{2}}, \quad h=\left(\phi_{w_{1}} \circ f\right) / \phi_{z_{1}} .
$$

By Schwarz-Pick Lemma 1.2, we have $g, h: \Delta \rightarrow \Delta$, and

$$
\begin{array}{ll}
g\left(z_{1}\right)=\frac{w_{2}-w_{1}}{z_{2}-z_{1}} \frac{1-\overline{z_{2}} z_{1}}{1-\overline{w_{2}} w_{1}}, & g\left(z_{2}\right)=f^{*}\left(z_{2}\right), \\
h\left(z_{2}\right)=\frac{w_{2}-w_{1}}{z_{2}-z_{1}} \frac{1-z_{2} \overline{z_{1}}}{1-w_{2} \overline{w_{1}}}, & h\left(z_{1}\right)=f^{*}\left(z_{1}\right) .
\end{array}
$$

The estimates in (A) give

$$
\begin{aligned}
\frac{\left|g\left(z_{1}\right)\right|-\left|\phi_{z_{1}}\left(z_{2}\right)\right|}{1-\left|g\left(z_{1}\right)\right|\left|\phi_{z_{1}}\left(z_{2}\right)\right|} \leq\left|g\left(z_{2}\right)\right| & \leq \frac{\left|g\left(z_{1}\right)\right|+\left|\phi_{z_{1}}\left(z_{2}\right)\right|}{1+\left|g\left(z_{1}\right)\right|\left|\phi_{z_{1}}\left(z_{2}\right)\right|}, \\
\text { that is, } \frac{\left|h\left(z_{2}\right)\right|-\left|\phi_{z_{1}}\left(z_{2}\right)\right|}{1-\left|h\left(z_{2}\right)\right|\left|\phi_{z_{1}}\left(z_{2}\right)\right|} \leq\left|g\left(z_{2}\right)\right| & \leq \frac{\left|h\left(z_{2}\right)\right|+\left|\phi_{z_{1}}\left(z_{2}\right)\right|}{1+\left|h\left(z_{2}\right)\right|\left|\phi_{z_{1}}\left(z_{2}\right)\right|} .
\end{aligned}
$$

Applying estimates (A) to $\left|h\left(z_{2}\right)\right|$ now (and observing (B)), we obtain the desired result.

Remark 2.2. If $f$ is not an automorphism, then we may apply the increasing function $t \mapsto(1 / 2) \log ((1+t) /(1-t))$ to either side of Proposition 2.1, and we use (C) and (D) to obtain

$$
\rho\left(f^{*}\left(z_{1}\right), 0\right)-2 \rho\left(z_{1}, z_{2}\right) \leq \rho\left(f^{*}\left(z_{2}\right), 0\right) \leq \rho\left(f^{*}\left(z_{1}\right), 0\right)+2 \rho\left(z_{1}, z_{2}\right),
$$

which is Theorem 1.3.

A more careful analysis yields a little more. With the same notation, we set

$$
\begin{aligned}
& \sigma_{1}=g\left(z_{1}\right)=\frac{w_{2}-w_{1}}{z_{2}-z_{1}} \frac{1-\overline{z_{2}} z_{1}}{1-\overline{w_{2}} w_{1}}, \\
& \sigma_{2}=h\left(z_{2}\right)=\frac{w_{2}-w_{1}}{z_{2}-z_{1}} \frac{1-z_{2} \overline{z_{1}}}{1-w_{2} \overline{w_{1}}},
\end{aligned}
$$


4 Schwarz-Pick-type estimates for the hyperbolic derivative

$p=\phi_{f *\left(z_{1}\right)} \circ g$, and $q=\phi_{\sigma_{1}} \circ h$. Here, estimates in (A) give

$$
\frac{\left|p\left(z_{1}\right)\right|-\left|\phi_{z_{1}}\left(z_{2}\right)\right|}{1-\left|p\left(z_{1}\right)\right|\left|\phi_{z_{1}}\left(z_{2}\right)\right|} \leq\left|p\left(z_{2}\right)\right| \leq \frac{\left|p\left(z_{1}\right)\right|+\left|\phi_{z_{1}}\left(z_{2}\right)\right|}{1+\left|p\left(z_{1}\right)\right|\left|\phi_{z_{1}}\left(z_{2}\right)\right|} \text {. }
$$

As before $\left|p\left(z_{1}\right)\right|=\left|q\left(z_{1}\right)\right|$, and applying (A) (and (B)) gives

$$
\begin{aligned}
\left|p\left(z_{2}\right)\right| & =\left|\phi_{f^{*}\left(z_{1}\right)}\left(f^{*}\left(z_{2}\right)\right)\right| \\
& \leq \frac{\left(\left|q\left(z_{2}\right)\right|+\left|\phi_{z_{1}}\left(z_{2}\right)\right|\right) /\left(1+\left|q\left(z_{2}\right)\right|\left|\phi_{z_{1}}\left(z_{2}\right)\right|\right)+\left|\phi_{z_{1}}\left(z_{2}\right)\right|}{1+\left(\left(\left|q\left(z_{2}\right)\right|+\left|\phi_{z_{1}}\left(z_{2}\right)\right|\right) /\left(1+\left|q\left(z_{2}\right)\right|\left|\phi_{z_{1}}\left(z_{2}\right)\right|\right)\right)\left|\phi_{z_{1}}\left(z_{2}\right)\right|} \\
& =\frac{\left(\left|\phi_{\sigma_{1}}\left(\sigma_{2}\right)\right|+\left|\phi_{z_{1}}\left(z_{2}\right)\right|\right) /\left(1+\left|\phi_{\sigma_{1}}\left(\sigma_{2}\right)\right|\left|\phi_{z_{1}}\left(z_{2}\right)\right|\right)+\left|\phi_{z_{1}}\left(z_{2}\right)\right|}{1+\left(\left(\left|\phi_{\sigma_{1}}\left(\sigma_{2}\right)\right|+\left|\phi_{z_{1}}\left(z_{2}\right)\right|\right) /\left(1+\left|\phi_{\sigma_{1}}\left(\sigma_{2}\right)\right|\left|\phi_{z_{1}}\left(z_{2}\right)\right|\right)\right)\left|\phi_{z_{1}}\left(z_{2}\right)\right|} .
\end{aligned}
$$

Likewise,

$$
\frac{\left(\left|\phi_{\sigma_{1}}\left(\sigma_{2}\right)\right|-\left|\phi_{z_{1}}\left(z_{2}\right)\right|\right) /\left(1-\left|\phi_{\sigma_{1}}\left(\sigma_{2}\right)\right|\left|\phi_{z_{1}}\left(z_{2}\right)\right|\right)-\left|\phi_{z_{1}}\left(z_{2}\right)\right|}{1-\left(\left(\left|\phi_{\sigma_{1}}\left(\sigma_{2}\right)\right|-\left|\phi_{z_{1}}\left(z_{2}\right)\right|\right) /\left(1-\left|\phi_{\sigma_{1}}\left(\sigma_{2}\right)\right|\left|\phi_{z_{1}}\left(z_{2}\right)\right|\right)\right)\left|\phi_{z_{1}}\left(z_{2}\right)\right|} \leq\left|\phi_{f^{*}\left(z_{1}\right)}\left(f^{*}\left(z_{2}\right)\right)\right|
$$

Again applying the increasing function $t \mapsto(1 / 2) \log ((1+t) /(1-t))$ when $f$ is not an automorphism, we obtain the following, which improves Theorem 1.4. (Having $z_{2}=0$ and requiring $f(0)=0$ yield $\sigma_{1}=\sigma_{2}$.)

Proposition 2.3. For $f: \Delta \rightarrow \Delta$ analytic and not an automorphism,

$$
\left|\rho\left(f^{*}\left(z_{1}\right), f^{*}\left(z_{2}\right)\right)-\rho\left(\sigma_{1}, \sigma_{2}\right)\right| \leq 2 \rho\left(z_{1}, z_{2}\right) \quad \forall z_{1}, z_{2} \in \Delta
$$

Remark 2.4. We cite [3], which contains various other generalizations of Theorem 1.4, one of which (Corollary 4.4) has conclusion

$$
\rho\left(\frac{1-z_{1} \overline{z_{2}}}{\overline{z_{1}} z_{2}-1} f^{*}\left(z_{1}\right), \frac{1-w_{1} \overline{w_{2}}}{\overline{w_{1}} w_{2}-1} f^{*}\left(z_{2}\right)\right) \leq 2 \rho\left(z_{1}, z_{2}\right) \quad \forall z_{1}, z_{2} \in \Delta
$$

([3] also contains some Euclidean versions, as does [5].)

\section{Other results}

Theorem 1.3 is obtained in [6] by integrating the following theorem.

Theorem 3.1 (see [6]). Let $f: \Delta \rightarrow \Delta$ be analytic. Then,

$$
\left|\frac{d}{d z}\right| f^{*}(z)|| \leq \frac{1-\left|f^{*}(z)\right|^{2}}{1-|z|^{2}}
$$

Below we refine this result using the same sort of procedure as above. (Then, in principle, a sharpening of Theorem 1.3 could be obtained via integration.) 
Proposition 3.2. Let $f: \Delta \rightarrow \Delta$ be analytic. Then,

$$
\left|\frac{d}{d z}\right| f^{*}(z)|| \leq \frac{\left|\phi_{f^{*}(z)}\left(\phi_{f(z)}(f(0)) / z\right)\right|+|z|^{2}}{|z|\left(1+\left|\phi_{f^{*}(z)}\left(\phi_{f(z)}(f(0)) / z\right)\right|\right)} \frac{1-\left|f^{*}(z)\right|^{2}}{1-|z|^{2}} .
$$

Proof. With $f$ as given, set

$$
g(\lambda)=\phi_{f(z)} \circ\left(f \circ \phi_{z}(\lambda)\right), \quad h(\lambda)=\phi_{g^{\prime}(0)}(g(\lambda) / \lambda) .
$$

Then $g(0)=0$, and so $h(0)=0$. We apply the upper estimate in (A) to $h(\lambda) / \lambda$, then have $\lambda \rightarrow 0$, to obtain

$$
\left|h^{\prime}(0)\right| \leq \frac{|h(z)|+|z|^{2}}{|z|(1+|h(z)|)}
$$

Now $h^{\prime}(0)=g^{\prime \prime}(0) / 2\left(\left|g^{\prime}(0)\right|^{2}-1\right)$, and so

$$
\frac{\left|g^{\prime \prime}(0)\right|}{2\left(1-\left|g^{\prime}(0)\right|^{2}\right)} \leq \frac{|h(z)|+|z|^{2}}{|z|(1+|h(z)|)}
$$

Here $g^{\prime}(0)=f^{*}(z)$, and a straightforward computation (cf. [6, Section 2]) reveals that

$$
\left|g^{\prime \prime}(0)\right|=2\left(1-|z|^{2}\right)\left|\frac{d}{d z}\right| f^{*}(z)||
$$

as desired.

Remarks 3.3. (i) Schwarz's Lemma 1.1 applied to $h$ gives $\left(\left|\phi_{f^{*}(z)}\left(\phi_{f(z)}(f(0)) / z\right)\right|+|z|^{2}\right)$ $/|z|\left(1+\left|\phi_{f^{*}(z)}\left(\phi_{f(z)}(f(0)) / z\right)\right|\right) \leq 1$, so this is indeed a refinement. (ii) The lower estimate in (A) would similarly yield a lower estimate for $|d / d z| f^{*}(z)||$. We leave the details to the reader. (iii) In [6], the author compares Theorem 3.1 with Schwarz-Pick Lemma 1.2. Proposition 3.2 may be similarly compared with Dieudonné's lemma (e.g., [2, 4]), which refines Schwarz-Pick Lemma 1.2. A perfect analog of Dieudonné's lemma would read $|d / d z| f^{*}(z)|| \leq\left(\left(\left|f^{*}(z)\right|+|z|^{2}\right) /|z|\left(1+\left|f^{*}(z)\right|\right)\right)\left(\left(1-\left|f^{*}(z)\right|^{2}\right) /\left(1-|z|^{2}\right)\right) \quad$ (for $\left.f^{*}(0)=0\right)$. However, this is not a refinement: for $f(\lambda)=\lambda^{2}$, we have $|d / d z| f^{*}(z)||=$ $\left(1-\left|f^{*}(z)\right|^{2}\right) /\left(1-|z|^{2}\right)$ but $\left(\left|f^{*}(z)\right|+|z|^{2}\right) /|z|\left(1+\left|f^{*}(z)\right|\right)=2$ when $z=0$. (At any $z$ for which $f(z)=f(0)$, we have $|h(z)|=\left|f^{*}(z)\right|$, so a perfect analog does occur at such points.)

\section{Acknowledgments}

The author is grateful to George T. Hole, his colleague in the Department of Philosophy, for bringing [1] to his attention, and to John Pfaltzgraff of The University of North Carolina at Chapel Hill for bringing [6] to his attention. 
6 Schwarz-Pick-type estimates for the hyperbolic derivative

\section{References}

[1] A. F. Beardon, The Schwarz-Pick lemma for derivatives, Proceedings of the American Mathematical Society 125 (1997), no. 11, 3255-3256.

[2] P. L. Duren, Univalent Functions, Fundamental Principles of Mathematical Sciences, vol. 259, Springer, New York, 1983.

[3] H. T. Kaptanoğlu, Some refined Schwarz-Pick lemmas, The Michigan Mathematical Journal 50 (2002), no. 3, 649-664.

[4] P. R. Mercer, Sharpened versions of the Schwarz lemma, Journal of Mathematical Analysis and Applications 205 (1997), no. 2, 508-511.

[5] __ Another look at Julia's lemma, Complex Variables 43 (2000), no. 2, 129-138.

[6] S. Yamashita, The Pick version of the Schwarz lemma and comparison of the Poincare densities, Annales Academiae Scientiarum Fennicae. Series A I. Mathematica 19 (1994), no. 2, 291-322.

Peter R. Mercer: Department of Mathematics, Buffalo State College, NY 14222, USA

E-mail address: mercerpr@math.buffalostate.edu 